\title{
HIV-1 Tat C Modulates Expression of miRNA-101 to Suppress VE-Cadherin in Human Brain Microvascular Endothelial Cells
}

\author{
Ritu Mishra and Sunit Kumar Singh \\ Laboratory of Neurovirology and Inflammation Biology, Council of Scientific and Industrial Research-Centre for Cellular and Molecular Biology, \\ Hyderabad-500007, India
}

HIV-1 infection leads to the development of HIV-associated neurological disorders. The HIV-1 Tat protein has been reported to exert an adverse effect on blood-brain barrier integrity and permeability. Perturbation in permeability is mainly caused by disruptions in adherens junctions and tight junction proteins. We have identified HIV-1 Tat C-induced disruption of VE-cadherin mediated by miRNA101 in human brain microvascular endothelial cells (BMVECs). HIV-1 Tat C increased the expression of miR-101, which led to downregulation of VE-cadherin. Overexpression of miR-101 resulted into the suppression of VE-cadherin. Inhibition of miR-101 by the miRNA inhibitor enhanced the expression of VE-cadherin. We have demonstrated that VE-cadherin is a direct target of miR-101 using a luciferase reporter assay, which showed that mutated VE-cadherin 3'UTR and miR-101 cotransfection did not change luciferase activity. By overexpression and knockdown of miR-101, we have demonstrated that the expression level of claudin-5 is governed by the expression of VE-cadherin. These findings demonstrate a novel mechanism for the regulation of barrier permeability by miR-101 via posttranscriptional regulation of VE-cadherin in human BMVECs exposed to the HIV-1 Tat C protein.

\section{Introduction}

The CNS is protected by the blood-brain barrier (BBB) and the blood-CSF barrier (Miller et al., 2012). The BBB is composed of human brain microvascular endothelial cells (BMVECs) supported by astrocytic end feet and pericytes (Reese and Karnovsky, 1967). The barrier properties of BMVECs are due to tight junction proteins (TJPs) and adherens junction proteins (AJPs) (Dejana et al., 2008).

VE-cadherin, an endothelial cell-specific transmembrane AJP, has been reported to be a master regulator of endothelial cell-cell adhesive interactions (Lampugnani et al., 1992). TJPs mainly include claudins, occludins, and zona occludens (ZO). VE-cadherin interacts with several signaling partners to coordinate endothelial growth, TJ organization, and permeability (Dejana and Giampietro, 2012). Therefore, VE-cadherin is an

Received 0ct. 10, 2012; revised Dec. 23, 2012; accepted Feb. 6, 2013.

Author contributions: R.M. and S.K.S. designed research; R.M. performed research; R.M. and S.K.S. analyzed data; S.K.S. wrote the paper.

This work was supported by the DBT Grant BT/PR10709/AGR/36/598/2008 and by the National Institutes of Health-sponsored AIDS international Training Programme. We thank Prof. Joan W Berman (Department of Pathology, Albert Einstein College of Medicine, New York) for support, help, and valuable suggestions and the kind gift of BMVECs for this study, Mr. Manish K. Johri for construction of the pET-21b-Tat B clone, Prof. Udaykumar Ranga (Jawaharlal Nehru Centre for Advanced Scientific Research, Bangalore, India) for assistance, and the director of the Centre for Cellular and Molecular Biology for assistance in the completion of this study.

The authors declare no competing financial interests.

Correspondence should be addressed to Sunit K. Singh, PhD, New R\&D Building, 1st Floor, Centre for Cellular and Molecular Biology (CCMB), Uppal Road, Hyderabad-500007, A.P., India. E-mail: sunitsingh@ccmb.res.in or sunitsingh2000@gmail.com.

DOI:10.1523/JNEUROSCI.4796-12.2013

Copyright $\odot 2013$ the authors $\quad 0270-6474 / 13 / 335992-09 \$ 15.00 / 0$ important protein for the establishment and maintenance of endothelial barrier integrity (Harris and Nelson, 2010).

HIV-1 crosses the BBB during the early course of infection and infects resident brain macrophages/microglia (GonzálezScarano and Martin-Garcia, 2005; Persidsky et al., 2006). HIV-1 Tat protein secretes out extracellularly from the productively infected cells and affects BMVECs adversely (Westendorp et al., 1995; Xiao et al., 2000; Strazza et al., 2011). Previous studies have shown the altered expression of TJ/AJ proteins in endothelial cells caused by the HIV-1 Tat protein (Mahajan et al., 2008; Zhong et al., 2008; Gandhi et al., 2009); however, the exact mechanisms involved in this alteration are not well understood (Xu et al., 2011). In total HIV-1 infections, clade $\mathrm{C}$ alone infects $>56 \%$ patients (Geretti, 2006). The clade-specific differences of HIV-1 Tat proteins were reflected in the differential induction of cytokines and chemokines (Gandhi et al., 2009), including differential suppression of IL-10 expression in monocytes (Wong et al., 2010). In human primary astrocytes, the expression and function of indoleamine-2,3-dioxygenase and the generation of the neurotoxin kynurenine are known to be modulated differently by clade B and C Tat proteins (Samikkannu et al., 2009). The differential roles of clade $\mathrm{B}$ and $\mathrm{C}$ Tat proteins have been highlighted in HIV-associated neurological disorders (Gandhi et al., 2010).

miRNAs are small RNA sequences 19-24 nucleotides in length that are reported to regulate $\sim 60 \%$ of the genes in the human genome (Bartel, 2009), mostly by binding through complementary binding sites located primarily at the 3'UTR of mRNAs. The HIV-1 Tat protein has been reported to modulate the expression pattern of cellular miRNAs in neuronal cells (Eletto et al., 2008). Recently, we have also reported that the 
HIV-1 Tat protein induces the expression level of miRNA-32 in human microglial cells and subsequently modulates the expression level of TRAF3 adaptor protein in microglial cells (Mishra et al., 2012). To determine the role of the HIV-1 Tat protein as a helper of HIV-1 in its process of neuroinvasion, we investigated the perturbation of miRNA in BMVECs by the HIV-1 Tat C protein. We have identified the downregulation of VE-cadherin, the master regulator of brain endothelial permeability, as a result of upregulation of miR-101 in response to HIV-1 Tat C.

\section{Materials and Methods}

Cell culture. Human brain primary microvascular endothelial cells (BMVECs) were obtained from Dr. Joan Berman (Department of pathology, Albert Einstein College of Medicine, New York) as a kind gift. BMVECs were grown in M199 medium supplemented with human endothelial growth factor (\#E07600; Sigma), bovine brain serum, human serum, newborn calf serum, ascorbic acid, heparin sulfate, and glutamine (Invitrogen). BMVECs were grown in culture flasks, coverslips, and polytetrafluoroethylene (PTFE) membranes coated with $0.2 \%$ gelatin. For dose-dependent assays and time point studies, BMVECs were seeded at a $1 \times 10^{5}$ cell density in six-well culture dishes and incubated for $48-72 \mathrm{~h}$ to achieve the optimal confluency. For the luciferase assay, HeLa cells were grown in DMEM (\#12100-046; Invitrogen) supplemented with $10 \%$ fetal bovine serum, $100 \mathrm{U}$ of penicillin, and $100 \mu \mathrm{g} / \mathrm{ml}$ streptomycin (\#10378016; Invitrogen). For the transactivation assay, CEM-GFP cells (obtained from the NIH AIDS Research and Reference Reagent Program) were grown in RPMI 1640 (\#23400-021; Invitrogen) supplemented with $10 \%$ fetal bovine serum (\#16000-044; Invitrogen), 2 mm glutamine, and $100 \mathrm{U} / \mathrm{ml}$ penicillin/streptomycin. Cultures were maintained at $37^{\circ} \mathrm{C}$ in a humidified chamber with a constant supply of $5 \% \mathrm{CO}_{2}$.

Expression and purification of HIV Tat protein. HIV-1 Tat C protein was expressed and purified as per our standardized protocol described previously (Mishra et al., 2012). The identity of recombinant Tat proteins was determined by Western blot analysis using anti-Tat antibody (\#4138; NIH AIDS Research and Reference Reagent Program). Finally, recombinant Tat $\mathrm{C}$ Protein was assayed for the endotoxin level using the limulus amebocyte lysate assay (\#50-647 U; Lonza) as per the manufacturer's protocol and was found to be $0.04 \mathrm{EU} / \mu \mathrm{g}$ of protein preparation, much below the acceptable limit of endotoxin level ( $1 \mathrm{EU} / \mu \mathrm{g}$ of protein). To confirm the biological activity of the recombinant Tat protein, we performed a transactivation assay as described previously (Mishra et al., 2012). CEM-GFP cells (\#3655; NIH AIDS Research and Reference Reagent Program); containing a stably integrated GFP gene under the control of subtype-B LTR were used for transactivation assay. Purified Tat C protein $(5 \mu \mathrm{g} / \mathrm{ml})$ was transfected with the ProteoJuice Protein Transfection Reagent (71281-3; Novagen), followed by visualization of GFP expressions under a fluorescence microscope (Axio Observer-A1; Carl Zeiss) after $24 \mathrm{~h}$ of transfection.

microRNA targets predictions. Bioinformatic prediction tools (Pictar, Target Scan 5.2, and MicroRNA.org) were used to identify the potential targets of miR-101. The target binding sites of miR-101 were found in the 3'UTR of VE-cadherin (human CDH5) transcripts using bioinformatics-based target prediction tools.

Tat treatment on BMVECs. In most of the assays, Tat $C$ had been exposed to BMVECs at $500 \mathrm{ng} / \mathrm{ml}$, a concentration chosen because of its closeness to the reported serum level of Tat protein that BMVECs would normally encounter in AIDS patients (Westendorp et al., 1995; Poggi et al., 2004) Human BMVECs were grown to confluency and exposed to HIV-1 Tat C protein at various doses $(100,200$, and $500 \mathrm{ng} / \mathrm{ml}, 1 \mu \mathrm{g} / \mathrm{ml}$, and $1.5 \mu \mathrm{g} / \mathrm{ml}$ ) in serum-free media. Control cells were treated with the Tat buffer (elution buffer/storage buffer of Tat protein). BMVECs were exposed to heat-inactivated Tat C (HI-Tat C; produced by heating at $85^{\circ} \mathrm{C}$ for $30 \mathrm{~min}$ ) as a control. After $12 \mathrm{~h}$ of HI-Tat C protein exposure, BMVECs were harvested for extraction of RNA and protein.

RNA isolation and miRNA assay. RNA isolation was done with miRNeasy kit (\#217004; Qiagen). cDNA synthesis for miRNA was done with miRNA-specific primers using the TaqMan reverse transcription kit
(\#4366596; Applied Biosystems). Thermal incubations were as follows; $16^{\circ} \mathrm{C}$ for $30 \mathrm{~min}, 42^{\circ} \mathrm{C}$ for $30 \mathrm{~min}$, and $85^{\circ} \mathrm{C}$ for $5 \mathrm{~min}$. miRNA assays were done by quantitative PCR ( $\mathrm{qPCR}$ ) using miRNA-specific TaqMan probes and the universal PCR master mix (\#4324018; Applied Biosystems). Thermal cycles were $95^{\circ} \mathrm{C}$ for $10 \mathrm{~min}$, followed by 40 cycles of $95^{\circ} \mathrm{C}$ for $15 \mathrm{~s}$ and $60^{\circ} \mathrm{C}$ for $60 \mathrm{~s}$. The thermal cycler ABI 7900 was used for all quantitative real-time PCR experiments.

Reverse transcription and real-time PCR. RNA was treated with DNase (\#M0303L; New England Biolabs) for $30 \mathrm{~min}$ at $37^{\circ} \mathrm{C}$. cDNA synthesis was done with Superscript II (\#11904-18; Invitrogen) as per the manufacturer's protocol. Thermal incubations were as follows: $65^{\circ} \mathrm{C}$ for $5 \mathrm{~min}$, $25^{\circ} \mathrm{C}$ for $10 \mathrm{~min}, 42^{\circ} \mathrm{C}$ for $50 \mathrm{~min}, 70^{\circ}$ for $10 \mathrm{~min}$, and, finally, RNase $\mathrm{H}$ treatment for $20 \mathrm{~min}$ at $37^{\circ} \mathrm{C}$. TaqMan assays were used for the detection of VE-cadherin transcripts (\#Hs00901463; Applied Biosystems).

Cell lysates and Western blot analysis. RIPA buffer (150 mM NaCl, 50 mм Tris- $\mathrm{HCl}, \mathrm{pH} 7.5,1 \% \mathrm{NP}-40,0.5 \%$ sodium doxycholate, $0.1 \%$ SDS, and $1 \times$ protease inhibitor mixture) was used for cell lysis. Protein concentrations were determined with the Bradford assay (\#500-0006; BioRad). Equal amounts of proteins were separated on $12 \%$ SDS gel and transferred on PVDF membrane at $100 \mathrm{~V}$ for $2 \mathrm{~h}$. Membranes were blocked in 5\% skim milk powder prepared in TBS-Tween 20 (TBST) buffer. Membranes were incubated overnight at $4^{\circ} \mathrm{C}$ with primary antibody (1:1000). After 3 washes of $10 \mathrm{~min}$ each with TBST, HRPconjugated secondary antibodies were applied for $45 \mathrm{~min}$. Membranes were again washed in TBST for 3 times and developed by using SuperSignal developing reagent (\#34095; Pierce). Antibodies against VEcadherin (\#ab76428; Abcam), anti-claudin-5 (\#34-1600; Invitrogen), anti-ZO-1 (\#61-7300; Invitrogen), anti-occludin (\#33-1520; Invitrogen), anti- $\beta$-tubulin (\#ab6046; Abcam), anti-Dicer (\#ab14601; Abcam), anti-Drosha (\#ab12286; Abcam), anti-TRBP (\#ab42018; Abcam), and anti-Ago2 (\#ab57113; Abcam) were used in this study.

miRNA overexpression. Before $1 \mathrm{~d}$ of the transfection experiment, BMVECs were seeded in six-well plates at $60 \%$ confluency. Transfection mixtures were prepared in Opti-MEM (\#11058-021; Invitrogen) and cells were kept in antibiotic-free media during transfection. miR-101 was overexpressed by transfecting the miRNA expression plasmid (\#SC400719; Origene) with Lipofectamine 2000 (\#11668-019; Invitrogen). Empty vector was used as a vehicle control. pCMV-mir-29b and scramble miR-101 were transfected into BMVECs as nonspecific controls. miR-101 overexpression was confirmed by qPCR using TaqMan probes specific to miR-101.

Anti-miR (miRNA inhibitor) transfection. BMVECs were transfected with 100 picomoles of anti-miR-101 (\#AM11414; Ambion) and Cy3labeled control anti-miR (\#AM17011; Ambion) with Lipofectamine 2000. Cells were pelleted for RNA isolation and protein lysate preparation after $48 \mathrm{~h}$ of transfection. Transfection efficiency was monitored by visualizing the fluorescence of Cy3-labeled control anti-miR. Knockdown of miR-101 in anti-miR-transfected cells was confirmed by miR101 assay. VE-cadherin protein expression was analyzed in BMVECs transfected with anti-miR-101 by Western blotting with anti-VEcadherin antibody.

Luciferase reporter assay. HeLa cells were seeded in six-well plates and cotransfected with luciferase reporter clones of CDH5 3'UTR and miR101-expressing plasmid using Lipofectamine 2000. The CDH5 3'UTR construct in pMirTarget (\#HmiT000052-MT01; GeneCopeia) and miR101 construct as pCMV-Mir (\#SC400013; Origene) were used in this assay. Complementary base sequences, CATGAC, from position 1566 to 1571 of the CDH5 3'UTR were deleted to abrogate the complementary binding between miR-101 and the VE-cadherin 3'UTR. A site-directed mutagenesis kit (\#200518; Stratagene) was used for generating the deletion mutations. Both the wild-type (WT) and mutant (MUT) 3'UTRs of $\mathrm{CDH} 5$ were transfected along with miR-101 expression clones in HeLa cells. Cells were harvested for luciferase assays after $24 \mathrm{~h}$ of transfection. Luciferase assays were performed with a luciferase assay kit (\#E4030; Promega) according to the manufacturer's protocol. The $\beta$-galactosidase assay (\#E2000; Promega) was used for normalization.

Transendothelial electrical resistance assay. A $3.0 \mu \mathrm{m}$ pore PTFE membrane insert (12-well plate; \#3494; Corning Life Sciences) was used in the permeability assays. EVOM2 (World Precision Instruments) was used to 
read the electrical resistance in all experiments. Insert membranes were precoated with $0.2 \%$ gelatin followed by cell seeding at a density of $2 \times 10^{4}$ in $500 \mu$ l of complete media in the upper chamber of a Transwell membrane. Additional gelatin coating over membrane inserts was found to be more helpful in optimal adherence of BMVECs rather than direct seeding of cells on collagen-coated surfaces. Transendothelial electrical resistance (TEER) values were monitored until the BMVECs reached optimal confluency $(\sim 4-6 \mathrm{~d})$. When TEER values became quite stable and were not showing any further increase, monolayers were considered as a confluent layer. Once BMVECs reached confluency in a monolayer, they were treated with different doses of Tat $C$ protein and TEER was recorded after $12 \mathrm{~h}$. TEER of BMVECs was recorded at different time points in a time course experiment.

Fluorescence permeability assay. BMVECs were seeded on a $3.0 \mu \mathrm{m}$ pore PTFE membrane insert similar to that described for the TEER assay. BMVECs were treated with different doses of Tat proteins and, after $12 \mathrm{~h}$ of treatment, cells were washed with HBSS buffer and incubated with $0.005 \%$ water-soluble sodium fluorescein (molecular weight 376.28; \#064738; Survival Research Laboratories) solution in the upper chamber of the insert membrane. After $30 \mathrm{~min}$ of incubation at $37^{\circ} \mathrm{C}, 100$ $\mu \mathrm{l}$ of the solution was collected from the lower chamber of wells and assayed for the fluorescence at 480/530 nm wavelength (Infinite M200; Tecon).

Statistical analysis. Data are expressed as the mean \pm SEM from three independent biologically repeated experiments. $p<0.05$ was considered as significant in a one-tailed array. Comparison of the treated group versus the untreated group was analyzed with Student's $t$ test. For the miR-101 assay, results are presented as the fold change relative to controls.

\section{Results}

miR-101 expression level increases upon HIV-1 Tat C treatment in human BMVECs

We reported previously that HIV-1 Tat C is able to induce the cellular level of certain miRNAs in human microglial cells (Mishra et al., 2012). Based on the available data about the role of the HIV-1 Tat protein in the modulation of cellular miRNAs, we hypothesized that there might be perturbations in miRNA expression patterns in BMVECs upon HIV-1 Tat exposure. In the present study, we investigated the expression level of miR101 in the Tat C-treated BMVECs to search for any perturbation due to the HIV-1 Tat C protein. Interestingly, the expression level of miR-101 in BMVECs showed a significant upregulation $(p \leq 0.005)$ after Tat $\mathrm{C}$ exposure in a dosedependent manner (Figure 1A). The expression level of miR-101 was $\sim 2.5$-fold higher compared with control cells when exposed to $100 \mathrm{ng} / \mathrm{ml}$ Tat $\mathrm{C}$ protein and this increase was linear, as shown in Figure $1 A$. To determine the specificity of the HIV-1 Tat C protein for induction of miR-101, we also investigated the level of miR-101 in BMVECs treated with the pET-21b empty-vectoreluted fraction, as well as with the HI-Tat $\mathrm{C}$ protein. The eluted empty vector $\mathrm{pET}-21 \mathrm{~b}$ did not result in the upregulation of miR101 (Fig. 1E). Similarly, HI-Tat C protein treatment of BMVECs
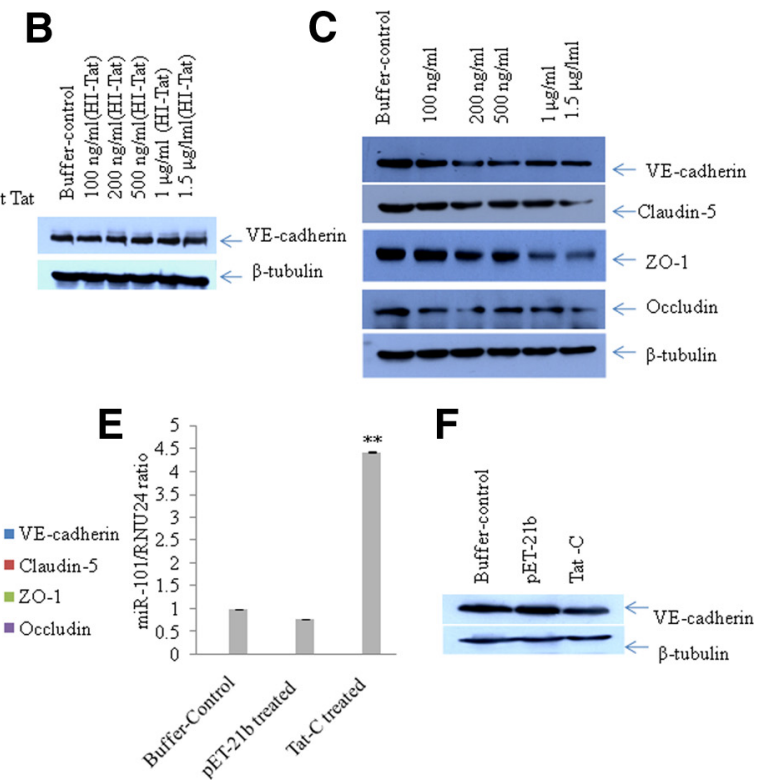

Figure 1. Expression of VE-cadherin and other TJPs decreases upon Tat $C$ treatment in a dose-dependent manner. $\boldsymbol{A}$, Fold change in miR-101 expression level after treatment with increasing doses of HIV-1 Tat C protein and HI-Tat C in BMVECs. BMVECs and VE-cadherin in BMVEC s exposed to the Tat C protein are statistically significant. ${ }^{* *} p \leq 0.005 \cdot{ }^{*} p \leq 0.05$. For $70-1{ }^{*} p \leq 0.05$ at all doses; at $1.5 \mu \mathrm{g} / \mathrm{ml}$ Tat C treatment, ${ }^{* *} p \leq 0.005$. For occludin, downregulation is statistically significant at all doses of Tat $p \leq 0.05$; at $1.5 \mu \mathrm{g} / \mathrm{ml}$ Tat C treatment, ${ }^{* *} p \leq 0.005$. Downregulation of claudin-5 was statistically significant. ${ }^{*} p \leq 0.05$ miR-101 expression level in BMVECS after Tat C treatment and empty vector PET-21b purified in a similar manner showing that upregulation of miR-101 is specific to Tat C. $\boldsymbol{F}$, Western blot analysis of the pET-21b and Tat C-treated BMVECs showing that downregulation of VE-cadherin is specific to Tat $C$ and is not induced by either buffer or empty vector. All experiments were repeated three times and are represented as mean $\pm \mathrm{SE}$.

did not affect the expression of miR-101 in dose-dependent manner (Fig. 1A).

HIV-1 Tat C exposure to BMVECs suppresses the expression of VE-cadherin and other TJPs

To study the effects of HIV-1 Tat C on BMVECs, we determined the expression levels of various TJPs (Claudin-5, ZO-1, occludin) and the major AJP VE-cadherin. We found a significant decrease in the expression of VE-cadherin and TJPs (Claudin-5, ZO-1, occludin) at the protein level in BMVECs exposed to Tat $\mathrm{C}$ in a dose-dependent manner (Fig. 1B). The downregulation of VE-cadherin was totally abrogated when BMVECs were treated with $\mathrm{HI}$-Tat $\mathrm{C}$ protein in similar doses (Fig. 1B). Treatment of BMVECs with empty pET-21b vector did not affect the expression of VE-cadherin (Fig. $1 F$ ). Not just increasing the dose of Tat $\mathrm{C}$, but also the extended duration of exposure $(2,6,12$, and $24 \mathrm{~h})$ of Tat $\mathrm{C}$ to BMVECs also resulted in a significant decrease in the expression level of AJP and VE-cadherin (Fig. $2 A, B$ ). In another experiment, BMVECs were exposed to Tat $B$ protein, and the VE-cadherin expression level did not decrease significantly compared with that of the Tat C protein (Fig. 3D). Remarkably, miR-101 was not upregulated in the BMVECs exposed to HIV Tat-B protein (Fig. $3 C$ ). These findings suggest that the regulation of VE- 
A

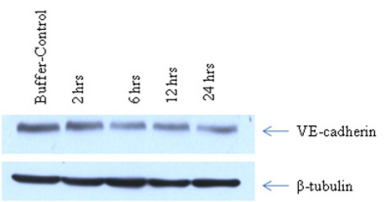

B
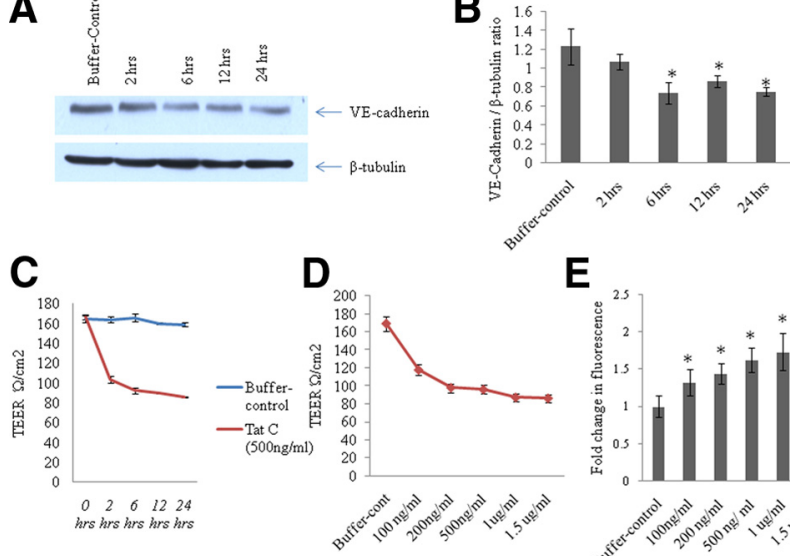

E

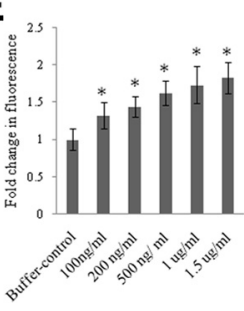

Figure 2. Decrease in expression of VE-cadherin and TEER with extended exposure of Tat $\mathrm{C}$ to BMVECs and disruption of endothelial barrier function. $A$, Western blot analysis showing decrease in the expression level of VE-cadherin in BMVECs with the increase in time of exposure of Tat C ( $500 \mathrm{ng} / \mathrm{ml})$ to BMVECs. B, Graph representative of three independent experiments showing a decrease in VE-cadherin expression with the increase in time of exposure of Tat C (500 $\mathrm{ng} / \mathrm{ml})$ to BMVECs. The VE-cadherin expression level started decreasing significantly after $6 \mathrm{~h}$ $(p \leq 0.05)$. C, Graph showing the decrease in TEER value with the increase in time of exposure of Tat $C(500 \mathrm{ng} / \mathrm{ml})$ to BMVECs. Increase in the time of exposure of the Tat C protein on BMVECs disrupted the barrier integrity of BMVECs and thereby increased the permeability $(p \leq 0.005)$. $\boldsymbol{D}$, Graph showing the dose-dependent decrease in the TEER value after Tat C treatment. $\boldsymbol{E}$, Bars indicating the fold changes in fluorescence after leakage of fluorescent compound (sodium fluorescein) across the monolayer of BMVECs exposed to Tat C protein $(p \leq 0.05)$. The migration of sodium fluorescein through control cells was set at 1 and the results are shown accordingly. As the dose of Tat $\mathrm{C}$ is increasing, the migration of sodium fluorescein is increasing from upper compartment to the lower compartment of the Transwell insert membrane culture experiment. All experiments were repeated three times and are represented as mean $\pm \mathrm{SE}$.
A
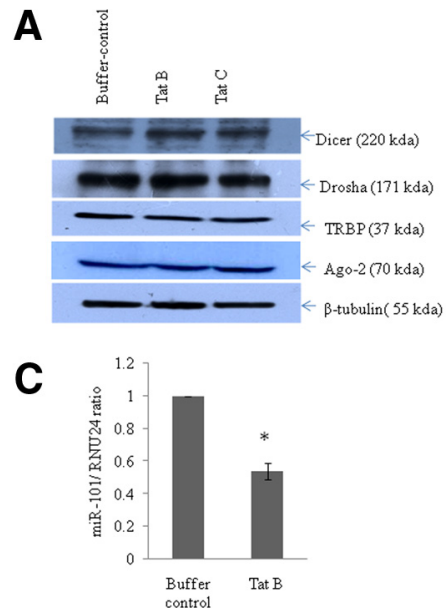

B

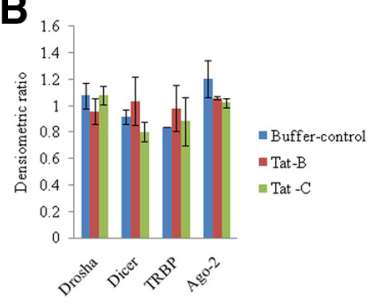

D

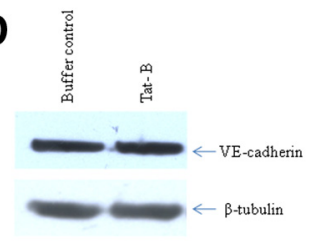

Figure 3. No significant effect of HIV-1 Tat $B$ or Tat $C$ on the expression of major miRNA biogenesis pathway proteins. $\boldsymbol{A}$, Western blot analysis for tracking the changes in expression level (if any) of major proteins involved in miRNA biogenesis. BMVECs were exposed for $12 \mathrm{~h}$ to Tat $B$ and Tat C proteins $(500 \mathrm{ng} / \mathrm{ml})$ and cells were harvested and tested for major proteins (Drosha, Dicer, Ago-2, TRBP). Neither clade of HIV-1 Tat protein (Tat B and Tat C) could exert any significant change in the expression of Drosha, Dicer, Ago-2, or TRBP. B, Densitometry quantitation of Drosha, Dicer, TRBP, and Ago-2 proteins as normalized with image density of $\beta$-tubulin by using ImageJ software. Results are representative of three independent biological repeats and are shown as mean \pm SE. No significant change can be seen $(p \geq 0.05)$. C, Bar diagram showing downregulation of miR-101 in BMVECs exposed to the Tat B protein. The expression level of miR-101 was quantified by specific TaqMan probes using RNU24 as a normalizer. $\boldsymbol{D}$, Western blot analysis of VE-cadherin in Tat B-treated BMVECs demonstrates the differential regulation of VE-cadherin expression. All experiments were repeated three times and are represented as mean $\pm S E$. cadherin in BMVECs is mediated posttranscriptionally by miR-101 induced by the HIV-1 Tat C protein.

HIV-1 Tat C treatment exerts endothelial barrier dysfunction Given the major role of AJPs and TJPs in the maintenance of barrier integrity in BMVECs, we also investigated the effect of the HIV-1 Tat C protein on barrier permeability. BMVECs grown on the insert membrane were treated with Tat C protein (100 ng to $1.5 \mu \mathrm{g}$ ) for the dose-dependent experiment and for the time points $2-24 \mathrm{~h}$ for the time course experiment, as described in the Materials and Methods. Tat $\mathrm{C}$ treatment resulted into a significant $(p \leq 0.005)$ decrease in TEER values for all doses of Tat $\mathrm{C}$ protein exposed to the BMVECs (Fig. $2 D$ ). TEER values also decreased significantly when measured at different exposure times (Fig. 2C). These results indicated that the downregulation of VE-cadherin and other TJPs (Fig. $1 C, D$, Fig. $2 A, B$ ) due to HIV-1 Tat $C$ treatment is responsible for the barrier disruption in BMVECs shown as decreased resistance across the endothelial monolayer. Leakage of a fluorescent substance (sodium fluorescein) was also used as a standard for determining the BMVEC permeability in this study. The migration of sodium fluorescein increased with the increase in the dose of Tat $\mathrm{C}$ exposure to BMVECs (Fig. 2E).

HIV-1 Tat C does not regulate miRNA biogenesis machinery Virus and viral products are known to modulate the cellular RNAi machinery in various ways (Santhakumar et al., 2010). To study whether the perturbation in the expression level of miR101 in BMVECs exposed to the HIV-1 Tat C protein is associated with any alteration in the miRNA biogenesis machinery, we determined the expression level of major proteins (Drosha, Dicer, Ago2, and TRBP) involved in miRNA biogenesis. In BMVECs exposed to HIV-1 Tat C and Tat B, we could not find any substantial change in the expression of these proteins (Fig. $3 A, B$ ). This observation indicated that the HIV-Tat protein does not modulate the expression of major proteins involved in miRNA biogenesis machinery in BMVECs.

miR-101 targets the 3'UTR of VE-cadherin directly

miR-101 was induced in BMVECs exposed to the HIV-1 Tat C protein (Fig. 1A). To identify the effect of miRNA-mediated posttranscriptional regulation of miR-101 in BMVEC permeability; we searched for potential targets of miR-101 using bioinformatics prediction tools (Targetscan 5.2, Pictar, and Miranda), which showed strong and multiple binding sites for miR-101 in the 3'UTR of VE-cadherin (also referred to as CDH5 3'UTR). As shown in Figure $1 A$, the dose-dependent increase in the expression of miR-101 after Tat C treatment and the concordant decrease in the expression level of VE-cadherin suggests that the expression level of VE-cadherin might be regulated by miR-101 (Fig. 4A). VE-cadherin 3'UTR is one of the longest $3^{\prime} \mathrm{UTR}$, which introduced the strong possibility of its posttranscriptional regulation being mediated by miRNAs. We used a luciferase reporter assay to determine whether miR-101 binds the VEcadherin 3'UTR directly. The construct encompassing firefly luciferase reporter followed by the full-length $3^{\prime} \mathrm{UTR}$ of VEcadherin was used. Expression constructs for miR-101, miR-29b, and empty vector were cotransfected with the VE-cadherin 3'UTR reporter into HeLa cells. The mutant 3'UTR construct of VE-cadherin (having deletion in binding sites complementary to miR-101; Fig. 4A) was also transfected to determine the specificity of the binding of miR-101 with the cadherin $3^{\prime}$ UTR. Luciferase activity was diminished by almost $65 \%$ after 


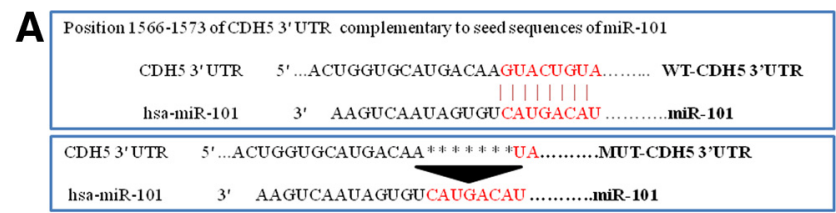

B

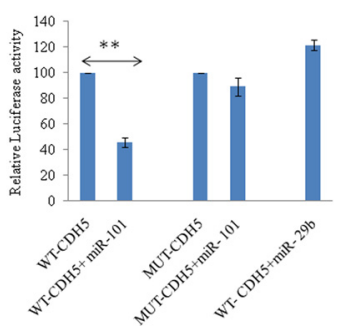

Figure 4. miR-101 binds complementary sequences in CDH5 3'UTR directly and regulates the expression of CDH5 (VE-cadherin). $\boldsymbol{A}$, Schematic representation of seed sequences in miR101 and complementary 8-mer binding sites in WT-CDH5 3'UTR (VE-cadherin). The complementary sites are deleted in MUT-CDH53'UTR using a site-directed mutagenesis kit to abrogate the regulatory interaction of miR-101 in the $3^{\prime}$ UTR of CDH5. WT and MUT-CDH5 $3^{\prime}$ UTR reporter constructs were cotransfected with miR-101, miR-29b, and pCMV- $\beta$-gal (as a normalizing control) plasmids and luciferase assays were performed in the HeLa cells. $B$, miR-101 transfection suppressed the luciferase activity significantly $(p \leq 0.005)$ in the WT-CDH53'UTR cotransfection. miR-101 transfection could not represse the luciferase activity when cotransfected with the MUT-CDH5 3'UTR. An irrelevant miR-29b, which does not have the complementary sequences for CDH5 3' UTR, did not perturb the luciferase activity. The luciferase expression level of cells (transfected with only 3'UTR of VE-cadherin) was considered as a control with $100 \%$ relative light unit and the results are shown accordingly. All experiments were repeated three times and are represented as mean \pm SE. ${ }^{* *} p \leq 0.005$.

cotransfection of the VE-cadherin $3^{\prime} \mathrm{UTR}$ and miR-101 $(p \leq$ 0.005 ; Fig. $4 B$ ). The mutant 3 'UTR construct of VE-cadherin (Fig. 4A) devoid of complementary sequences for miR-101 did not show significant reduction in luciferase activity (Fig. $4 B$ ). The cotransfection of 3'UTR of VE-cadherin and an irrelevant miR$29 \mathrm{~b}$ expression construct also did not show any decrease in the luciferase activity (Fig. 4B).

miR-101 overexpression downregulates cellular VE-cadherin expression level

After confirming the site-specific complementary binding between miR-101 and VE-cadherin 3'UTR, we investigated the effect of miR-101 at the protein expression level of VE-cadherin in BMVECs. A P-CMV-miR-101 construct encoding miR-101 was transfected in BMVECs, and the overexpression of miR 101 was confirmed by determining the level of expression of miR-101 using the TaqMan assay. After $24 \mathrm{~h}$ of transfection, the level of expression of miR-101 was nearly 8.5 -fold higher (Fig. $5 A$; $p<$ $0.05)$. Overexpression of miR-101 resulted into a $45 \%$ reduction of VE-cadherin expression at the protein level (Fig. $5 B, C$ ). The specificity of regulation was determined by transfecting another irrelevant miRNA, miR-29b (Fig. 5B,D). This miRNA was not able to suppress the expression level of VE-cadherin in BMVECs (Fig. $5 B, C$ ). The reduction was also evident at the mRNA level of VE-cadherin in miR-101-transfected cells (Fig. 5D). These results demonstrated that the cellular expression level of miR-101 has an important role in the translational regulation of VE-cadherin. As a proof of concept, the scramble miR-101 was also transfected in BMVECs at 100 and 200 pmol concentrations. The scramble miR-101 sequence did not bring about any change in the expression level of VE-cadherin (Fig. 5F).
Anti-miR-101 rescues the expression level of VE-cadherin in the presence of $\mathrm{HIV}-1$ Tat $\mathrm{C}$ protein

The objective of this experiment was to determine both the regulatory effect of miR-101 on VE-cadherin expression and to determine the rescuing ability of anti-miR-101 in preserving the expression level of VE-cadherin even in the presence of HIV-1 Tat C, which has been shown to suppress VE-cadherin and thereby the permeability of BMVECs. To confirm the direct regulation of expression of VE-cadherin by miR-101, we tranfected the BMVECs with the miRNA inhibitor of miR-101, here referred to as anti-miR-101. After transfection of anti-miR-101, BMVECs were exposed to the HIV-1 Tat C protein. Cy3-labeled scramble anti-miR was used to test the transfection efficiency and as a negative control. The cellular miR-101 levels were quantified using $\mathrm{qPCR}$ to confirm the modulation of miR-101 as per the experimental design (Fig. 6A). The stand-alone transfection of anti-miR-101 in BMVECs was found to upregulate the expression level of VE-cadherin significantly (Fig. $6 B ; p \leq 0.05$ ). However, when the miR-101-transfected cells were additionally treated with the HIV-1 Tat C protein, there was a significant regain of VE-cadherin expression (Fig. $6 B, C$; $p \leq 0.05$ ), indicating that anti-miR-101 was able to nullify the suppressive effect of Tat $\mathrm{C}$ on the expression of VE-cadherin mediated by miR-101.

\section{Expression level of VE-cadherin influences the expression level of Claudin-5 directly}

VE-cadherin and claudin-5 represent the key components of the AJs and TJs, respectively (Nitta et al., 2003). HIV-1 Tat C exposure to BMVECs showed a gradual, dose-dependent decrease in the expression levels of both proteins (Fig. $1 B$ ) and a decrease in the permeability of BMVECs (Fig. 2D). The expression level of claudin-5 was also monitored in the experimental setups using overexpression of miR-101 and anti-miR-101 transfection, in which we had already showed that the regulation of VE-cadherin expression is mediated by miR-101. The rationale behind these experiments was to demonstrate the regulatory role of VEcadherin over claudin-5 in BMVECs. The upstream regulatory role of VE-cadherin on claudin-5 has also been reported previously (Taddei et al., 2008). A regulatory role of VE-cadherin on claudin- 5 suggested that VE-cadherin might play a central role in maintaining the endothelial cell lineage, which was again supported by the fact that claudin-5 expression is limited to the VE-cadherin-expressing cells (Gavard and Gutkind, 2008).

In all experiments, we observed a trend showing a regulatory role of VE-cadherin on claudin-5 (Fig. 7A). Upon overexpression of miR-101, when VE-cadherin was being suppressed, we observed the same trend for claudin-5 (Fig. 7C). In BMVECs transfected with anti-miR-101, there was a significant regain of claudin-5 expression (Fig. $7 B, D$ ), which is consistent with the regain of VE-cadherin in anti-miR-101 transfection (Fig. 6C, Fig. 8 ). These results confirm the idea of functional hierarchy of VE-cadherin in the establishment of the endothelial cell-cell junctions.

\section{Discussion}

The BBB acts as a structural and functional barrier protecting the CNS from macroconstituents such as drugs, pathogens, and neurotoxins flowing from the periphery to the CNS and vice versa (Spudich and Ances, 2011). Many neurotropic viruses, such as HIV-1, human T-cell leukemia virus (HTLV-1), lymphocytic choriomeningitis virus (LCMV), West Nile virus (WNV), and hantaviruses, are known to disrupt the $\mathrm{BBB}$ via the endothelial junctions (Spindler and Hsu, 2012). HIV-1 infection in the CNS 
A

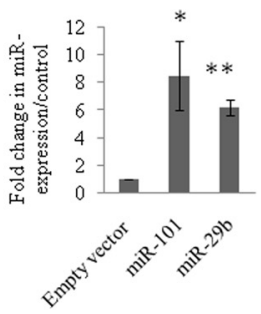

B
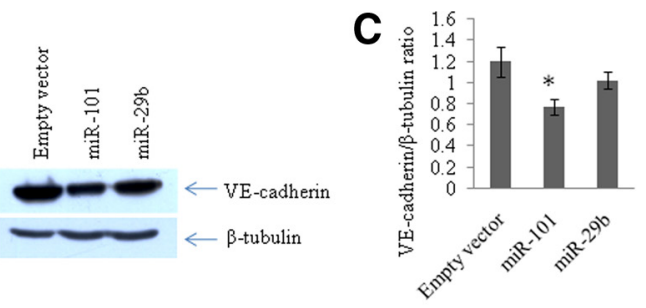

D

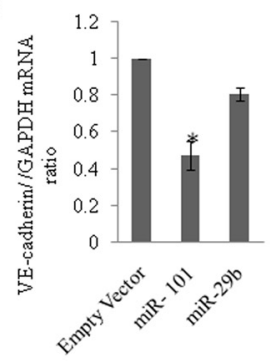

E

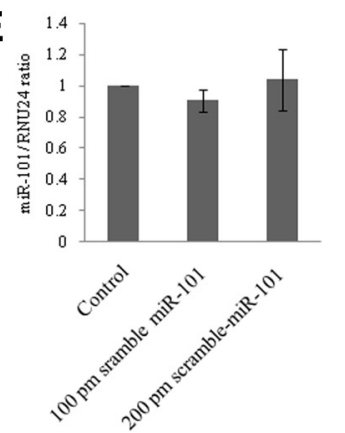

$\mathbf{F}$

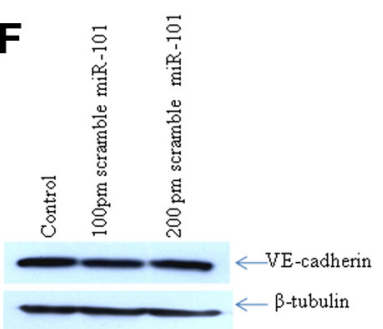

Figure 5. Overexpression of miR-101 reduces the expression of VE-cadherin in BMVECs. $\boldsymbol{A}$, Graph showing the fold change in miR-101 and miR 29b in miR-101- and miR-29b-overexpressed BMVECs, respectively, via qPCR using the TaqMan miR-101 and TaqMan miR-29b assays. The miR-101 level was found to be 8.5-fold higher in miR-101-transfected cells compared with vector control $(p \leq 0.05)$. B. Western blot analysis for VE-cadherin in BMVECs after overexpression of miR-101. Empty vector was used as a negative control and the plasmid pCMV-miR-29b was used as nonspecific miRNA to test the specificity of regulation. In miR-101 overexpression, the VE-cadherin expression level is downregulated significantly $(p \leq 0.05)$. C, Densitometry image density analysis of VE-cadherin using $\beta$-tubulin as a normalizer in ImageJ software. All experiments were repeated three times and are represented as mean \pm SE. D, qPCR analysis of VE-cadherin in miR-101- and miR-29b-overexpressed cells to determine changes at the transcript level. Total RNA was reverse transcribed by using random hexamers and transcript levels were quantified with VE-cadherin-specific TaqMan probes using GAPDH as an internal reference. Fold changes were determined with the $\Delta \Delta \mathrm{ct}$ method. $\boldsymbol{E}$, Fold change in miR-101 expression level after transfection of scramble miR-101 in BMVECs showing no significant change. $\boldsymbol{F}$, Western blot analysis for VE-cadherin in scramble miR-101-transfected BMVECs showing no change in their protein expression level. All experiments were repeated three times and are represented as mean \pm SE.

is reported to be an early event after primary infection (Hazleton et al., 2010), however, the mechanisms of perturbation of the BBB during HIV-1 infection are not fully understood. The HIV-1 Tat protein has been implicated in HIV neuropathogenesis and is regarded as a major player in BBB disruption (Hui et al., 2012). HIV-1 Tat has been reported as an active player in several vascular pathologies of HIV-1 in the CNS, in which HIV does not infect the cell directly, but affects the functionality in a bystander fashion (Zhong et al., 2008). The circulating Tat protein in the serum of HIV-1-infected patients has been reported to range from 300 to 550 ng/ml (Westendorp et al., 1995; Poggi et al., 2004). To mimic the conditions of this bystander effect, we performed our experiments on the primary human BMVECs by exposing them to the HIV-1 Tat C protein. We demonstrated that HIV-1 Tat C upregulates the expression levels of $\mathrm{miR}$ 101 in BMVECs (Fig. 1A), which in turn posttranscriptionally regulate the expression level of VE-cadherin. Pacifici et al. (2013) reported the induced expression of miR-101 in their miRNA expression profiling of the CSF of AIDS patients displaying the symptoms of $\mathrm{HIV}$-associated encephalitis. It is known that the HIV-1 Tat protein can easily transfuse through cells (Frankel and Pabo, 1988).

We found a significant upregulation of miR-101 in BMVECs on Tat C treatment. The modulation in the expression pattern of miRNAs has been reported in other cells exposed to HIV-1 Tat. Perturbation of the presynaptic protein SNAP25 via miR-128 (Eletto et al., 2008), suppression of the CYP2E1 protein in neurons through miR-1 (Fiore et al., 2009), and the induction of miR-34a to downregulate their target genes (Chang et al., 2011) have been described previously. We have also reported previously the miR-32mediated regulation of TRAF3 after Tat C exposure (Mishra et al., 2012). Having conserved seed sequences across the mammalian species, miR-101 seemed to have a potential role in gene regulation (John et al., 2006).

We found a dose-dependent decreased expression of a major AJP, VE-cadherin, in response to Tat $\mathrm{C}$ treatment. VE-cadherin has a predominant role in orchestrating the architecture of adherence junctions in endothelial cells. However, the precise mechanism for the regulation of VE-cadherin and its impact on endothelial permeability was not well understood, specifically in the case of the bystander hit of the HIV-1 Tat protein. This decrease in the expression of VE-cadherin was manifested at the level of endothelial functions in terms of the compromised permeability of the endothelial monolayer (Fig. 2C-E). Significant decreases in TEER value and accelerated fluorescein migration were observed even at low concentrations of Tat C $(100 \mathrm{ng} / \mathrm{ml})$, whereas the total VE-cadherin expression level did not change significantly. This might have been due to early inflammatory responses (secretion of cytokines, chemokines) in endothelial cells exposed to Tat, which could induce the internalization of
Figure 6. Anti-miR-101 transfection rescues the VE-cadherin expression level in BMVECs. $\boldsymbol{A}$, Anti-miR-101 transfection resulted in the reduced expression of miR-101. An $\sim 80 \%$ reduction in the expression of cellular miR-101 in anti-miR-101-transfected BMVECs was seen compared with scramble-transfected negative controls. ${ }^{*} p \leq 0.05$. $\boldsymbol{B}$, Western blot analysis to determine the regain in level of expression of VE-cadherin in anti-miR-101-transfected BMVECs. After $24 \mathrm{~h}$ of anti-miR-101 transfection in BMVECs, cells were again exposed to Tat C protein $(500 \mathrm{ng} / \mathrm{ml})$ to test the specificity of the miR-101-mediated control over the expression of VE-cadherin, but the expression of VE-cadherin remained unaffected due to the suppressive effect of anti-miR101 on miR-101 ( $p \leq 0.05$ ). C, Western blot images of VE-cadherin were normalized with $\beta$-tubulin and are presented as graph bars. All experiments were repeated three times and are represented as mean $\pm S E$. * $p \leq 0.05$. 

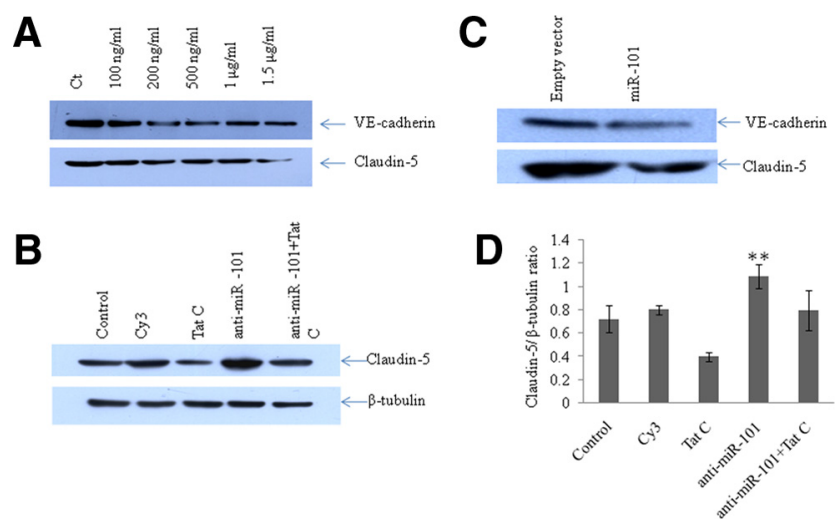

Figure 7. Expression level of VE-cadherin influences the expression level of Claudin-5 directly. To distinguish the direct effect of expression level of VE-cadherin on the expression level of claudin-5, three sets of experiments were performed. $A$, The downregulation of VE-cadherin upon Tat $C$ treatment decreases the expression level of claudin- 5 in a dose-dependent manner. $B$, Transfection of anti-miR-101 in BMVECs showing the same trend of rescued expression of claudin- 5 as in the regained expression of VE-cadherin, even in the presence of the Tat C protein. C, Overexpression of miR-101 decreases the expression level of claudin-5 in the same way as that of VE-cadherin. $D$, Densitometry analysis using ImageJ software of Western blot images for claudin-5 in anti-miR-101 transfection experiments. A significant recovery of claudin-5 expression level can be seen $(p \leq 0.005)$. All experiments were repeated three times and are represented as mean \pm SE. ${ }^{* *} p \leq 0.005$.

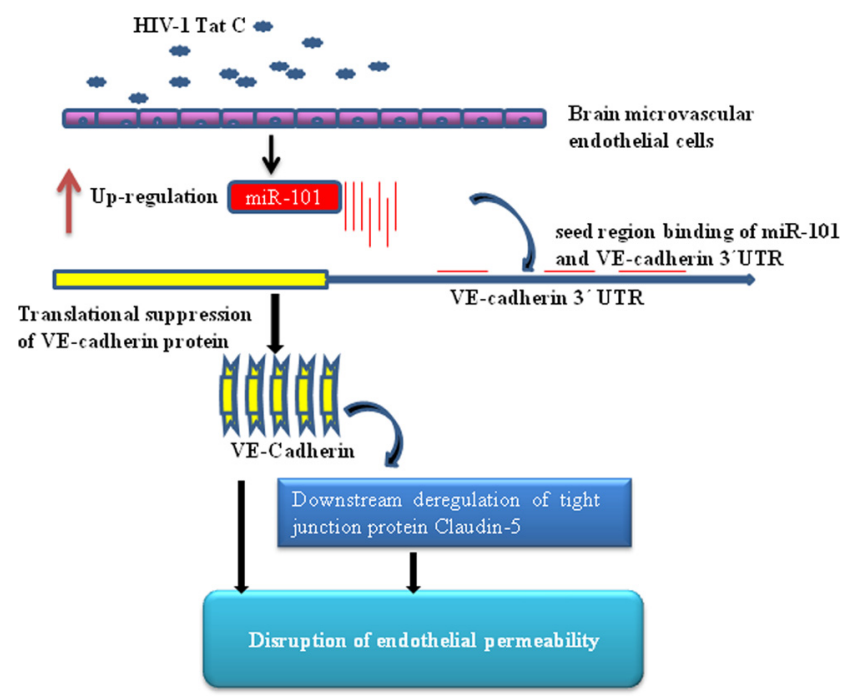

Figure 8. Proposed model for Tat C-mediated downregulation of VE-cadherin and claudin-5 in BMVECs through miR-101. We propose that expression of VE-cadherin is regulated through miR-101 in BMVECs exposed to the HIV-1 Tat C protein. After HIV-1 Tat C treatment, the expression level of miR-101 increases and targets the expression level of VE-cadherin directly, which in turn influences the expression level of claudin-5 and thereby the permeability in BMVECS.

VE-cadherin from the cell surface to the cellular compartment (Zhu et al., 2012).

Other groups have reported the disruption of TJs such as claudin-5, occludin, and ZO-1 (Zhong et al., 2008; András and Toborek, 2011; Xu et al., 2012) due to the effect of inflammatory cytokines/chemokines (Andriopoulou et al., 1999). Tat is reported to decrease occludin expression by cleaving through MMP9 (Xu et al., 2012). The downregulation of occludin and $\mathrm{ZO}-1$ in brain microvessels has been reported to be due to prostaglandin E2 (Pu et al., 2007). Activation of VEGFR-2 and many other redox-regulated signal transduction pathways are thought to contribute to the Tat-mediated downregulation of claudin-5 (András et al., 2005).
In this study, we elucidated the mechanism of downregulation of VE-cadherin, the predominant AJP. VE-cadherin is located on cell-cell junctions and primarily controls the physical adherence between cell membranes, along with connecting the intercellular network of cytoplasmic proteins such as p120, $\beta$-catenin, and plakoglobin and cytoskeletal microfilaments (Dejana et al., 1999; Vincent et al., 2004; Dejana et al., 2008). The expression level of VE-cadherin has been found frequently to be altered in inflammatory conditions such as diabetes and viral infections (Alexander et al., 2000; Dewi et al., 2008). We have demonstrated previously that HIV-1 Tat C is able to upregulate the expression of miR-101, which is accompanied by a decrease of VE-cadherin in BMVECs (Fig. 1). Overexpression of miR-101 in BMVECs resulted into the decreased expression of VE-cadherin at both the protein and mRNA levels (Fig. $5 B, D$ ). This was the first indication of VEcadherin regulation through miR-101. However, we did not observe reduced expression of VE-cadherin in the BMVECs transfected with irrelevant miR-29b (Fig. 5B), suggesting that the VE-cadherin regulation through miR-101 is highly specific. Scramble miR-101 was also transfected in BMVECs at 100 and 200 pmol concentrations. The cells transfected with scramble miR-101 showed perturbation in neither the expression of cellular miR-101 nor protein expression of VE-cadherin (Fig. 5E,F). This demonstrated the regulation of VE-cadherin through sequence-specific binding of seed sequences of miR-101 and 3'UTR of VE-cadherin.

To demonstrate the miRNA-mediated regulation more specifically, BMVECs were transfected with anti-miR-101. This inhibitor has the complementary sequences for the miR-101 seed region. The purpose of this transfection was to capture and restrict the expression level of cellular mature miR-101. As expected, it restrained the miR-101 level in BMVECs (Fig. 6A), which resulted in the elevated expression of VE-cadherin (Fig. $6 B)$. This regain of VE-cadherin became more obvious when BMVECs were transfected with anti-miR-101 and exposed to HIV-1 Tat C protein. Even in the presence of Tat C, BMVECs did not show downregulation of VE-cadherin (Fig. 6B). This revealed the potential effect of anti-miR-101 in combating the endothelial disruption. This finding confirmed that HIV-1 Tat C-mediated upregulation of miR-101, which is nullified by presence of antimiR-101 in transfected BMVECs, was now not able to reduce the VE-cadherin expression. This indicated that miRNA-mediated regulation may operate over and/or along with other mechanisms in the regulation of VE-cadherin in human BMVECs.

We performed a luciferase reporter assay to investigate the negative regulation of VE-cadherin by miR-101. Cotransfection of miR-101 with the 3'UTR of VE-cadherin suppressed the luciferase activity significantly $(p \leq 0.005)$. The luciferase activity was restored after mutating the binding sites of miR-101 in the 3'UTR luciferase reporter construct, which established VEcadherin as a direct target of miR-101 and explained the miR101-mediated posttranscriptional regulation of VE-cadherin in BMVECs. Specificity of the interaction between miR-101 and VE-cadherin was again demonstrated by the cotransfection of 3'UTR reporter constructs with the irrelevant miRNA miR-29b, which did not reduce the luciferase activity (Fig. 4B).

The brain's endothelial permeability is the joint attribute of both TJPs and AJPs. These proteins act together for maintaining the adhesive interactions required for barrier integrity of BMVECs. We have demonstrated that the posttranscriptional regulation of VE-cadherin is mediated through miR-101 and that the expression of VE-cadherin governs the expression patterns of the TJP claudin-5 directly. In experiments related to the overexpres- 
sion of miR-101 and suppression of cellular miR-101 by antimiR-101, the expression level of claudin-5 followed the same trend as that of VE-cadherin (Fig. $7 B, C$ ). These findings are consistent with previous studies showing that the levels of VEcadherin modulated the expression of claudin-5 directly (Taddei et al., 2008). VE-cadherin has been reported to control the transcription of claudin- 5 by tethering the repressive transcription factors FoxO1 away from the claudin-5 promoter (Taddei et al., 2008). In another study, Nitta et al. (2003) reported that VEcadherin-deficient mice were embryonically lethal compared with claudin-5 knock-out mice, which followed the course of normal development initially but died soon due to BBB dysfunction. These reports suggested that VE-cadherin plays a predominant regulatory role in the constitution of the endothelial barrier junctions in BMVECs. VE-cadherin is positioned upstream in the regulatory cascade and influences claudin-5 and the maintenance of endothelial barrier functions directly. The interactions of these two important proteins are unique to the brain barrier compared with other junctions such as epithelial cell junctions (Dejana, 2004). This master regulatory role of VE-cadherin provides another opportunity to modulate other downstream targets, playing an important role in vascular biology by shaping VE-cadherin expression in endothelial cells (Gavard and Gutkind, 2008). In the present study, we have demonstrated for the first time that HIV-1 Tat C upregulates the expression level of miR-101 in BMVECs and consequently suppresses the expression of VE-cadherin. The suppression of VE-cadherin results in the reduced expression level of claudin-5, which in turn compromises the permeability of BMVECs. This study presents a novel miRNA-mediated mechanism for the HIV-1 Tat C-induced decrease in VE-cadherin expression and subsequent dysfunctions in the barrier integrity of BMVECs.

\section{References}

Alexander JS, Alexander BC, Eppihimer LA, Goodyear N, Haque R, Davis CP, Kalogeris TJ, Carden DL, Zhu YN, Kevil CG (2000) Inflammatory mediators induce sequestration of VE-cadherin in cultured human endothelial cells. Inflammation 24:99-113. CrossRef Medline

András IE, Toborek M (2011) HIV-1-induced alterations of claudin-5 expression at the blood-brain barrier level. Methods Mol Biol 762:355-370. CrossRef Medline

András IE, Pu H, Tian J, Deli MA, Nath A, Hennig B, Toborek M (2005) Signaling mechanisms of HIV-1 Tat-induced alterations of claudin-5 expression in brain endothelial cells. J Cereb Blood Flow Metab 25: 1159-1170. CrossRef Medline

Andriopoulou P, Navarro P, Zanetti A, Lampugnani MG, Dejana E (1999) Histamine induces tyrosine phosphorylation of endothelial cell-to-cell adherens junctions. Arterioscler Thromb Vasc Biol 19:2286-2297. CrossRef Medline

Bartel DP (2009) MicroRNAs: target recognition and regulatory functions. Cell 136:215-233. CrossRef Medline

Chang JR, Mukerjee R, Bagashev A, Del Valle L, Chabrashvili T, Hawkins BJ, He JJ, Sawaya BE (2011) HIV-1 Tat protein promotes neuronal dysfunction through disruption of microRNAs. J Biol Chem 286:41125-41134. CrossRef Medline

Dejana E (2004) Endothelial cell-cell junctions: happy together. Nat Rev Mol Cell Biol 5:261-270. CrossRef Medline

Dejana E, Giampietro C (2012) Vascular endothelial-cadherin and vascular stability. Curr Opin Hematol 19:218-223. CrossRef Medline

Dejana E, Bazzoni G, Lampugnani MG (1999) Vascular endothelial (VE)cadherin: only an intercellular glue? Exp Cell Res 252:13-19. CrossRef Medline

Dejana E, Orsenigo F, Lampugnani MG (2008) The role of adherens junctions and VE-cadherin in the control of vascular permeability. J Cell Sci 121:2115-2122. CrossRef Medline

Dewi BE, Takasaki T, Kurane I (2008) Peripheral blood mononuclear cells increase the permeability of dengue virus-infected endothelial cells in association with downregulation of vascular endothelial cadherin. J Gen Virol 89:642-652. CrossRef Medline
Eletto D, Russo G, Passiatore G, Del Valle L, Giordano A, Khalili K, Gualco E, Peruzzi F (2008) Inhibition of SNAP25 expression by HIV-1 Tat involves the activity of mir-128a. J Cell Physiol 216:764-770. CrossRef Medline

Fiore R, Khudayberdiev S, Christensen M, Siegel G, Flavell SW, Kim TK, Greenberg ME, Schratt G (2009) Mef2-mediated transcription of the miR379-410 cluster regulates activity-dependent dendritogenesis by fine-tuning Pumilio2 protein levels. EMBO J 28:697-710. CrossRef Medline

Frankel AD, Pabo CO (1988) Cellular uptake of the tat protein from human immunodeficiency virus. Cell 55:1189-1193. CrossRef Medline

Gandhi N, Saiyed Z, Thangavel S, Rodriguez J, Rao KV, Nair MP (2009) Differential effects of HIV type 1 clade B and clade C Tat protein on expression of proinflammatory and antiinflammatory cytokines by primary monocytes. AIDS Res Hum Retroviruses 25:691-699. CrossRef Medline

Gandhi N, Saiyed ZM, Napuri J, Samikkannu T, Reddy PV, Agudelo M, Khatavkar P, Saxena SK, Nair MP (2010) Interactive role of human immunodeficiency virus type 1 (HIV-1) clade-specific Tat protein and cocaine in blood-brain barrier dysfunction: implications for HIV-1associated neurocognitive disorder. J Neurovirol 16:294-305. CrossRef Medline

Gavard J, Gutkind JS (2008) VE-cadherin and claudin-5: it takes two to tango. Nat Cell Biol 10:883-885. CrossRef Medline

Geretti AM (2006) HIV-1 subtypes: epidemiology and significance for HIV management. Curr Opin Infect Dis 19:1-7. CrossRef Medline

González-Scarano F, Martín-García J (2005) The neuropathogenesis of AIDS. Nat Rev Immunol 5:69-81. CrossRef Medline

Harris ES, Nelson WJ (2010) VE-cadherin: at the front, center, and sides of endothelial cell organization and function. Curr Opin Cell Biol 22:651-658. CrossRef Medline

Hazleton JE, Berman JW, Eugenin EA (2010) Novel mechanisms of central nervous system damage in HIV infection. HIV AIDS (Auckl) 2:39-49.

Hui L, Chen X, Bhatt D, Geiger NH, Rosenberger TA, Haughey NJ, Masino SA, Geiger JD (2012) Ketone bodies protection against HIV-1 Tatinduced neurotoxicity. J Neurochem 122:382-391. CrossRef Medline

John B, Sander C, Marks DS (2006) Prediction of human microRNA targets. Methods Mol Biol 342:101-113. Medline

Lampugnani MG, Resnati M, Raiteri M, Pigott R, Pisacane A, Houen G, Ruco LP, Dejana E (1992) A novel endothelial-specific membrane protein is a marker of cell-cell contacts. J Cell Biol 118:1511-1522. CrossRef Medline

Mahajan SD, Aalinkeel R, Sykes DE, Reynolds JL, Bindukumar B, Fernandez SF, Chawda R, Shanahan TC, Schwartz SA (2008) Tight junction regulation by morphine and HIV-1 tat modulates blood-brain barrier permeability. J Clin Immunol 28:528-541. CrossRef Medline

Miller F, Afonso PV, Gessain A, Ceccaldi PE (2012) Blood-brain barrier and retroviral infections. Virulence 3:222-229. CrossRef Medline

Mishra R, Chhatbar C, Singh SK (2012) HIV-1 Tat C-mediated regulation of tumor necrosis factor receptor-associated factor-3 by microRNA 32 in human microglia. J Neuroinflammation 9:131. CrossRef Medline

Nitta T, Hata M, Gotoh S, Seo Y, Sasaki H, Hashimoto N, Furuse M, Tsukita S (2003) Size-selective loosening of the blood-brain barrier in claudin5-deficient mice. J Cell Biol 161:653-660. CrossRef Medline

Pacifici M, Delbue S, Ferrante P, Jeansonne D, Kadri F, Nelson S, VelascoGonzalez C, Zabaleta J, Peruzzi F (2013) Cerebrospinal fluid miRNA profile in HIV-encephalitis. J Cell Physiol 228:1070-1075. CrossRef Medline

Persidsky Y, Heilman D, Haorah J, Zelivyanskaya M, Persidsky R, Weber GA, Shimokawa H, Kaibuchi K, Ikezu T (2006) Rho-mediated regulation of tight junctions during monocyte migration across the blood-brain barrier in HIV-1 encephalitis (HIVE). Blood 107:4770-4780. CrossRef Medline

Poggi A, Carosio R, Fenoglio D, Brenci S, Murdaca G, Setti M, Indiveri F, Scabini S, Ferrero E, Zocchi MR (2004) Migration of V delta 1 and V delta $2 \mathrm{~T}$ cells in response to CXCR3 and CXCR4 ligands in healthy donors and HIV-1-infected patients: competition by HIV-1 Tat. Blood 103:2205-2213. CrossRef Medline

Pu H, Hayashi K, András IE, Eum SY, Hennig B, Toborek M (2007) Limited role of COX-2 in HIV Tat-induced alterations of tight junction protein expression and disruption of the blood-brain barrier. Brain Res 1184: 333-344. CrossRef Medline

Reese TS, Karnovsky MJ (1967) Fine structural localization of a blood-brain 
barrier to exogenous peroxidase. J Cell Biol 34:207-217. CrossRef Medline

Samikkannu T, Saiyed ZM, Rao KV, Babu DK, Rodriguez JW, Papuashvili MN, Nair MP (2009) Differential regulation of indoleamine-2,3dioxygenase (IDO) by HIV type 1 clade B and C Tat protein. AIDS Res Hum Retroviruses 25:329-335. CrossRef Medline

Santhakumar D, Forster T, Laqtom NN, Fragkoudis R, Dickinson P, AbreuGoodger C, Manakov SA, Choudhury NR, Griffiths SJ, Vermeulen A, Enright AJ, Dutia B, Kohl A, Ghazal P, Buck AH (2010) Combined agonist-antagonist genome-wide functional screening identifies broadly active antiviral microRNAs. Proc Natl Acad Sci U S A 107:13830-13835. CrossRef Medline

Spindler KR, Hsu TH (2012) Viral disruption of the blood-brain barrier. Trends Microbiol 20:282-290. CrossRef Medline

Spudich SS, Ances BM (2011) Central nervous system complications of HIV infection. Top Antivir Med 19:48-57. Medline

Strazza M, Pirrone V, Wigdahl B, Nonnemacher MR (2011) Breaking down the barrier: the effects of HIV-1 on the blood-brain barrier. Brain Res 1399:96-115. CrossRef Medline

Taddei A, Giampietro C, Conti A, Orsenigo F, Breviario F, Pirazzoli V, Potente M, Daly C, Dimmeler S, Dejana E (2008) Endothelial adherens junctions control tight junctions by VE-cadherin-mediated upregulation of claudin-5. Nat Cell Biol 10:923-934. CrossRef Medline

Vincent PA, Xiao K, Buckley KM, Kowalczyk AP (2004) VE-cadherin: ad- hesion at arm's length. Am J Physiol Cell Physiol 286:C987-C997. Medline

Westendorp MO, Frank R, Ochsenbauer C, Stricker K, Dhein J, Walczak H, Debatin KM, Krammer PH (1995) Sensitization of T cells to CD95mediated apoptosis by HIV-1 Tat and gp120. Nature 375:497-500. CrossRef Medline

Wong JK, Campbell GR, Spector SA (2010) Differential induction of interleukin-10 in monocytes by HIV-1 clade B and clade C Tat proteins. J Biol Chem 285:18319-18325. CrossRef Medline

Xiao H, Neuveut C, Tiffany HL, Benkirane M, Rich EA, Murphy PM, Jeang KT (2000) Selective CXCR4 antagonism by Tat: implications for in vivo expansion of coreceptor use by HIV-1. Proc Natl Acad Sci U S A 97: 11466-11471. CrossRef Medline

Xu R, Feng X, Xie X, Zhang J, Wu D, Xu L (2012) HIV-1 Tat protein increases the permeability of brain endothelial cells by both inhibiting occludin expression and cleaving occludin via matrix metalloproteinase-9. Brain Res 1436:13-19. CrossRef Medline

Zhong Y, Smart EJ, Weksler B, Couraud PO, Hennig B, Toborek M (2008) Caveolin-1 regulates human immunodeficiency virus-1 Tat-induced alterations of tight junction protein expression via modulation of the Ras signaling. J Neurosci 28:7788-7796. CrossRef Medline

Zhu W, London NR, Gibson CC, Davis CT, Tong Z, Sorensen LK, Shi DS, Guo J, Smith MC, Grossmann AH, Thomas KR, Li DY (2012) Interleukin receptor activates a MYD88-ARNO-ARF6 cascade to disrupt vascular stability. Nature 492:252-255. CrossRef Medline 